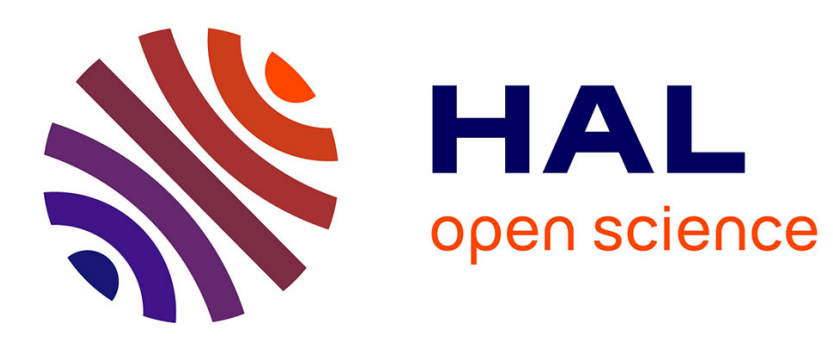

\title{
Contribution des éléments de gestion des réclamations à la satisfaction: les apports de la théorie de la justice
} William Sabadie, Isabelle Prim-Allaz, Sylvie Llosa

\section{To cite this version:}

William Sabadie, Isabelle Prim-Allaz, Sylvie Llosa. Contribution des éléments de gestion des réclamations à la satisfaction: les apports de la théorie de la justice. 22ème Congrès Intrenational de l'Association Française du Marketing, 2006, Nantes, France. pp.CD Rom. hal-00519837

\section{HAL Id: hal-00519837 \\ https://hal.science/hal-00519837}

Submitted on 21 Sep 2010

HAL is a multi-disciplinary open access archive for the deposit and dissemination of scientific research documents, whether they are published or not. The documents may come from teaching and research institutions in France or abroad, or from public or private research centers.
L'archive ouverte pluridisciplinaire HAL, est destinée au dépôt et à la diffusion de documents scientifiques de niveau recherche, publiés ou non, émanant des établissements d'enseignement et de recherche français ou étrangers, des laboratoires publics ou privés. 


\section{Contribution des éléments de gestion des réclamations à la satisfaction : les apports de la théorie de la justice}

Sabadie W., maître de conférences à l'IAE - Université de Lyon 3, équipe IRIS, centre de recherche Magellan, (sabadie@univ-lyon3.fr).

Prim-Allaz I., maître de conférences à l'IUT B - ISFA Université de Lyon 1 (Isabelle.Allaz@univ-lyon1.fr).

Llosa S., professeur des universités, Université Jean Monnet Saint Etienne, PREACTIS et Université Paul Cézanne Aix-Marseille, CEROG. 


\section{Contribution des éléments de gestion des réclamations à la satisfaction : les apports de la théorie de la justice}

Résumé :

La gestion des réclamations revêt une importance toute particulière dans le secteur des services. Cette recherche se propose de présenter les apports des théories de la justice pour la gestion des réclamations dans le cadre d'une expérience de service. Le modèle tétraclasse est mobilisé afin d'étudier la contribution des éléments de justice à la satisfaction.

Cette étude montre que, dans le cadre des relations entre les professionnels de santé et les Caisses Primaires d'Assurances Maladies, il existe effectivement différentes logiques de contribution des éléments de gestion des réclamations. Elle souligne que ces dernières sont caractéristiques selon que les éléments relèvent de la dimension distributive, procédurale ou interactionnelle de la justice.

Mots clés : satisfaction, gestion des réclamations, théorie de la justice, contribution, modèle tétraclasse.

\section{The contribution of service recovery components to satisfaction : An analysis through the justice theory}

\section{$\underline{\text { Abstract : }}$}

This research aims to propose a presentation of the justice theory contributions to the customer complaint handling in the context of a service experience. The Tetraclass model of satisfaction helps in defining the different contributions of the justice components.

This study indicates that in the context of relationships between health practitioners and the French social insurance, there are different contribution logics of the complaint handling elements.

Key Words : satisfaction, complaint handling, justice theory, responsibility, contribution, Tetra class model. 


\section{Contribution des éléments de gestion des réclamations à la satisfaction : les apports de la théorie de la justice}

La gestion des réclamations s'inscrit dans une démarche commerciale relationnelle visant à accroître la satisfaction, l'engagement et la confiance des clients de l'entreprise et à améliorer la qualité des prestations. Cette gestion revêt une importance toute particulière dans le secteur des services où la prestation est à la fois difficile à évaluer pour le client et délicate à contrôler pour l'entreprise. Dans le cadre de relations de service, l'importance du facteur humain, les échanges interpersonnels toujours différents et impossibles à formaliser totalement expliquent les nombreux incidents susceptibles de survenir. Le prestataire ne pouvant éviter complètement ces incidents de service, il se doit de prévenir les dysfonctionnements, mais aussi de prévoir un dispositif de gestion des réclamations efficace (Rust et al., 1996). La gestion des réclamations s'inscrit ainsi parfaitement dans les programmes de management de la qualité (Chébat et Slusarczyk, 2005) et offre de réelles opportunités pour retenir les clients ayant eu des problèmes avec leur prestataire de service, à condition qu'ils soient satisfaits du traitement de leur réclamation (Hart et al., 1990). Des chercheurs suggèrent que la réponse aux incidents peut renforcer la relation entre l'entreprise et le client (Blodgett et al., 1997 ; Maxham et Netemeyer, 2002b).

Le processus de réclamation implique des «transactions » entre le client mécontent et le prestataire mis en cause et ces échanges peuvent conduire à des mécanismes de compensation répondant plus ou moins aux attentes du client (Chébat et Slusarczyk, 2005). Une réclamation est caractérisée par trois éléments: l'émetteur, le motif et l'origine du problème. La réclamation peut être définie comme une demande d'information, de rectification et/ou de compensation, provenant du client et à destination de l'organisation, faisant suite à une insatisfaction, impliquant la responsabilité de l'organisation perçue par le client. Le champ inclut les réclamations amicales, entendues comme des propositions d'améliorations formulées vers l'organisation (co-auteurs, 2005). La réclamation appartient à un ensemble de réponses à l'insatisfaction perçue (Crié, 2001). Dans la présente étude, elle est considérée comme relevant d'une action explicite d'un client, ou de ses représentants, vers l'organisation concernée. Elle se distingue donc des actions qui ne sont pas orientées directement vers l'entreprise (bouche-à-oreille, plainte auprès d'associations de consommateurs ou de tribunaux, report des achats vers d'autres fournisseurs, par exemple). Cette distinction est 
importante car elle souligne l'intérêt stratégique d'un dispositif incitatif de collecte et de gestion des réclamations. Ce dernier doit permettre, d'un point de vue managérial, de prévenir et de réduire les risques liés aux ruptures de relations clients, de tirer les leçons de ses erreurs et de participer à l'amélioration de la profitabilité de l'entreprise (Bowen et al., 1999). Enfin, l'action de réclamation suppose que le client attribue tout ou partie de la responsabilité de son insatisfaction au fournisseur (Chébat et al., 2005). Cette attribution de la responsabilité incombe au client et c'est ce point de vue qui est décisif dans la gestion des réclamations. En effet, même si l'entreprise n'est pas responsable, mais que le client est convaincu du contraire, il attendra une gestion du problème au regard de la responsabilité qu'il attribue à l'entreprise. Ainsi, l'identification de l'origine de l'incident, telle que perçue par le client, est un enjeu majeur du processus de gestion des réclamations.

De nombreux travaux ont mobilisé les théories de la justice pour étudier les éléments contributifs à la satisfaction des clients confrontés à un incident de service (Blodgett et al., 1993 ; Hocutt et al., 1997 ; Tax et al., 1998 ; Smith et al., 1999 ; Sabadie, 2000 ; Maxham et Netemeyer 2002b; Tissot et Chandon, 2004). Les théories de la justice caractérisent la réparation en trois dimensions : distributive, procédurale et interactionnelle. Ces théories sont pertinentes pour plusieurs raisons. D'une part, il existe une promesse implicite de justice dans les services car il est souvent difficile pour le consommateur d'évaluer la prestation avant et parfois après l'avoir expérimenté (Seiders et Berry, 1998). Ainsi, un incident de service rompt les engagements implicites ou explicites pris par le prestataire. D'autre part, la prestation de service met souvent en jeu des relations interpersonnelles de nature coopérative. La relation de service implique que les employés et les clients co-produisent le service. Les travaux en psychologie sociale montrent l'intérêt de considérer le sentiment de justice des individus pour faciliter leur coopération. Par exemple, Thibaut et Walker (1975) suggèrent que l'utilisation de procédures regardées comme justes facilitent le maintien de relations positives parmi les membres d'un groupe. Elles préservent l'unité de ce groupe même dans les cas de conflits d'intérêts.

Si la littérature étudie la nature des éléments de gestion des réclamations qui contribuent à la satisfaction du client confronté à un incident de service, elle s'intéresse plus rarement aux poids relatifs de ces éléments dans cette satisfaction ce qui permettrait pourtant de hiérarchiser les priorités d'actions. Cet article met l'accent sur ce point. Par conséquent, son objectif est d'étudier la contribution des éléments de gestion des réclamations à la satisfaction des clients 
en intégrant l'idée, largement admise aujourd'hui, selon laquelle ces contributions ne sont pas linéaires (Anderson, 1973 ; Woodruff et al. 1983 ; Llosa, 1997).

Des éléments de réponses aux questions suivantes sont, ainsi, proposés :

- La contribution des éléments de gestion des réclamations à la satisfaction du client vis-à-vis de l'incident de service suit-elle différentes logiques? Existe-t-il des éléments qui influencent le niveau de satisfaction seulement lorsqu'ils sont perçus de façon positive? ou négative? Existe-t-il d'autres éléments qui jouent sur la satisfaction quelle que soit leur performance perçue?

- Le mode de contribution est-il différent et caractéristique selon que les éléments relèvent de la justice distributive, procédurale ou interactionnelle?

Pour répondre à ces questions une revue de la littérature sur le champ des réclamations souligne, dans un premier temps, l'intérêt des théories de la justice. Les travaux considérant la non-linéarité de la contribution des éléments de service à la satisfaction client sont ensuite mobilisés. Ceci conduit à la présentation du modèle tétra-classe de Llosa (1997) et à son application dans le cadre des relations entre des Caisses Primaires d'Assurance Maladie et les professionnels de santé.

\section{LES APPORTS DES THEORIES DE LA JUSTICE POUR LA GESTION DES RECLAMATIONS}

Les chercheurs en marketing utilisent assez largement la théorie de la justice dans le champ des traitements des problèmes survenus lors d'une rencontre de service (Blodgett et al., 1993 ; Tax et al., 1998 ; Smith et al., 1999 ; co-auteur, 2000 ; Maxham et Netemeyer 2002b ; Tissot et Chandon, 2004). En cohérence avec les travaux sur les rencontres de services, les recherches sur le concept de justice ont souligné le rôle joué par le résultat, le processus et l'interaction entre les individus (Folger et Cropanzano, 1998). L'idée de justice correspond à l'évaluation du fait que la réponse apportée par une partie à une autre est, ou non, appropriée compte tenu du préjudice causé par l'incident de service. La théorie de la justice met en exergue trois dimensions : distributive, procédurale et interactionnelle.

La dimension distributive concerne le jugement, par le client, de la réponse apportée par l'entreprise par rapport à ce que l'individu pense mériter. La démarche de réclamation vise à rétablir l'équilibre de l'échange entre un client, potentiellement déçu par l'incident de service, et l'entreprise qui n'a pas fourni une prestation à la hauteur de ce qui était attendu/annoncé. 
Le jugement du client repose sur une évaluation de l'équité perçue (Adams, 1965). L'équité peut être décrite comme un jugement en deux étapes. Dans la première étape, l'individu compare ses « contributions » à ses « avantages » (ou bénéfices) obtenus. Il a le sentiment d'être traité avec équité s'il perçoit que les avantages retirés sont justes au regard de ses contributions. C'est le principe de l'équité interne. La justice distributive perçue dépend également de la perception qu'a l'individu du ratio contributions/avantages d'autres personnes ou d'autres expériences prises en référence. L'individu a le sentiment d'être traité avec équité s'il perçoit que les avantages reçus, relativement à ses contributions, sont proportionnels aux avantages et contributions des personnes ou des expériences avec lesquelles il fait sa comparaison. C'est le principe de l'équité externe.

La dimension procédurale concerne les méthodes utilisées par le prestataire de service pour recevoir et traiter la réclamation. L'importance des éléments procéduraux tels que l'accessibilité, la simplicité du processus de réclamation ou la vitesse de réponse tient au fait que le client doit subir les pertes liées aux mauvaises performances du prestataire mais aussi celles liées aux efforts nécessaires pour résoudre ce problème.

Enfin, la dimension interactionnelle de la justice s'intéresse à la relation entre le client et le personnel en contact. On peut distinguer deux types d'éléments de justice interactionnelle (Folger et Cropanzano, 1998) : ceux liés à la décision ou au problème (les explications et les justifications) et ceux qui montrent un certain respect de la personne durant la procédure (politesse, empathie, bienveillance).

Les éléments de justice procédurale et interactionnelle contribuent à réduire le sentiment de frustration et de colère des clients. Ils participent à diminuer l'impact d'une décision qui n'est pas favorable au client (c'est-à-dire son sentiment d'un défaut de justice distributive suite au refus d'un échange ou d'un remboursement par exemple). C'est ce que Folger et Konovsky (1989) qualifient de «fair process effect» (cf. Lind et Tyler, 1988, pour une revue). Par exemple, dans un contexte organisationnel, Folger et Konovsky (1989) montrent que les employés pensant que leur directeur a évalué leurs performances d'une manière juste ont tendance à avoir des niveaux supérieurs de satisfaction vis-à-vis du salaire, de loyauté à leur organisation et de confiance dans le directeur, indépendamment du montant de salaire reçu. En d'autres termes, dans un contexte commercial, une entreprise qui écoute ses clients mécontents, reconnaît le problème en s'excusant, explique son origine et justifie la manière dont elle y répond, aurait plus de chance de les satisfaire, indépendamment des éléments distributifs. 
Un certain nombre de travaux se sont attachés à étudier l'influence des éléments de justice sur la satisfaction des clients. Cependant, comme le font remarquer Blodgett et al. (1997) ou encore Chébat et Slusarczyk (2005), peu de recherches ont permis de mettre en lumière un cadre théorique satisfaisant pour mieux comprendre comment chacune des trois facettes de la théorie de la justice influence l'évaluation de la gestion de la réclamation et les comportements post-réclamation. La hiérarchisation de l'importance des dimensions de la justice fait encore débat. Smith et al. (1999) et Maxham et Netemeyer (2002b) montrent que la dimension distributive est la plus importante pour expliquer la satisfaction des clients vis-àvis de l'entreprise, leurs intentions d'achat et de bouche-à-oreille. Les stratégies distributives concernent notamment l'assistance, le remboursement et les compensations. Les stratégies procédurales et interactionnelles se focalisent sur la manière dont l'entreprise définit ce qu'elle doit proposer suite à un incident de service. La plupart des travaux soulignent notamment l'importance de la rapidité de réponse et de l'attitude du personnel en contact. (Blodgett et al.,1993 ; Hart et al., 1990 ; Maxham et Netemeyer, 2002b ; Smith et al., 1999 ; Tax et al., 1998). L'importance des éléments procéduraux tels que l'accessibilité, la simplicité du processus de réclamation ou la vitesse de réponse tient au fait que le client doit subir les pertes liées aux mauvaises performances du prestataire mais aussi celles liées aux efforts nécessaires pour résoudre ce problème. Tax et al. (1998) montrent que la dimension interactionnelle est la plus importante pour expliquer la satisfaction vis-à-vis d'un processus de gestion des réclamations ${ }^{1}$. Les capacités d'écoute et d'empathie du personnel en contact contribuent à réduire le sentiment de frustration et de colère des clients. Des travaux récents (Boshoff et Leong, 1998; Maxham et Netemeyer, 2003) soulignent l'impact du comportement du personnel en contact, au-delà des procédures formalisées par l'entreprise (« extra-role behaviors » ou «empowerment »), sur la justice perçue des clients. Lorsque les procédures de gestion des incidents ne sont pas trop formalisées, le personnel en contact a la possibilité d'évaluer la situation et de s'adapter au contexte en proposant une stratégie de réponse spécifique (Boshoff et Leong, 1998 ; Maxham et Netemeyer, 2002a). Cependant, aucun de ces travaux ne s'est véritablement focalisé sur la contribution des éléments de gestion des réclamations selon le niveau de performance du prestataire. Seuls Chébat et Slusarczyk (2005) montrent que dans le cas de la justice procédurale, la lenteur du traitement

\footnotetext{
${ }^{1}$ Ces résultats semblent en contradiction avec ceux proposés par Smith et al. (1999), toutefois, Tax et al. (1998) étudient la satisfaction vis-à-vis de la gestion de la réclamation tandis que Smith et al. (1999) s'intéressent à la satisfaction vis-à-vis de l'entreprise suite à un incident de service.
} 
de la réclamation entraîne des émotions négatives alors que la rapidité de traitement est associée à des émotions positives. Nous proposons d'étudier plus avant cette non linéarité des contributions au moyen des travaux de Llosa (1997).

\section{LES MODES DE CONTRIBUTION DES ELEMENTS DE GESTION DES RECLAMATIONS A LA SATISFACTION}

Pour la majorité des chercheurs, la satisfaction est conceptualisée comme un continuum unidimensionnel opposant deux pôles extrêmes: positif (très satisfait) et négatif (très insatisfait) - Howard et Sheth, 1969 ; Nicosia, 1971 ; Oliver, 1980 ; Woodruff et al., 1983 ; Westbrook, 1987 -. Le paradigme de confirmation/infirmation des attentes, abondamment présent dans la littérature sur la "satisfaction" suppose ainsi que le poids d'un élément dans la satisfaction du client est prédéfini et demeure constant quelle que soit la façon dont se déroule l'expérience de service (logique de poids fixe). Cependant, ce processus comporte des limites sérieuses telles que la remise en cause de standards de comparaisons toujours pré-établis (Kahneman et Miller 1986; Cadotte et al. 1987 ; Carman, 1990 ; Iacobucci et al., 1994 ; Grönroos,1993), la présence de zone d'indifférence (Woodruff et al., 1983) ou la présence d'effet de seuil (Schneider et Bowen, 1995). Ces limites suggèrent que certains éléments peuvent contribuer à la satisfaction du client différemment selon leur niveau de performance perçue (logique de poids variables). Cette idée est renforcée par d'autres courants de recherche : d'une part, la littérature sur les "processus de choix" ouvre le champ de réflexion aux modèles d'agrégation non-compensatoires (Myers et Alpert, 1968 ; Dubois, 1980 ; Vernette, 1986). D'autre part, les recherches initiées en 1959 par Herzberg et al. concernant la satisfaction dans le travail apportent un éclairage radicalement différent sur la construction de la satisfaction, plaidant pour l'existence de poids dépendant du niveau de performance. Des auteurs en marketing, influencés par ces derniers travaux, considèrent que la satisfaction est bi-dimensionnelle (Swan et Combs, 1976; Maddox, 1981; Silvestro et Johnston, 1992 ; Smith et al., 1992) : les facteurs qui engendrent la satisfaction sont différents et indépendants de ceux qui engendrent l'insatisfaction ${ }^{2}$. Une troisième catégorie de chercheurs réconcilie ces deux visions : la satisfaction est unidimensionnelle, mais la fonction de contribution de

\footnotetext{
${ }^{2}$ La plupart d'entre eux ajoutent aux facteurs de satisfaction et aux facteurs d'insatisfaction d'autres catégories d'éléments : des facteurs "hybrides" jouant fortement sur la satisfaction et l'insatisfaction ou des facteurs "neutres" ayant toujours un faible impact, quelle que soit leur évaluation par le client.
} 
certains éléments à la satisfaction est non linéaire. Les méthodes de simulations de Kano et al. (1984), de "pénalité/récompense" de Brandt (1988, 1998), des deux importances de Venkitaraman et Jaworski (1993), le modèle «tétraclasse» de Llosa (1997) ou celui d'Audrain (2004) vont dans ce sens.

Le modèle "tétraclasse" possède en particulier une validité externe solide puisque l'existence de ces quatre types d'éléments n'a pas été infirmé dans diverses recherches fondées sur des activités de services très variées (Llosa, 1997; Bartikowski, 1999; Lichtlé et al., 2002 ; Clerfeuille et Poubanne, 2002; Léo et Philippe, 2003 ; Poubanne et al., 2003 ; Ray et Gotteland, 2004 ; Merdinger-Rumpler, 2004 ; Bartikowski et Llosa, 2004). Il propose deux modes principaux de contribution, chacun se divisant en deux modes secondaires (Cf. figure 1) :

Figure 1- Les différentes logiques de contribution à la satisfaction du modèle Tétraclasse

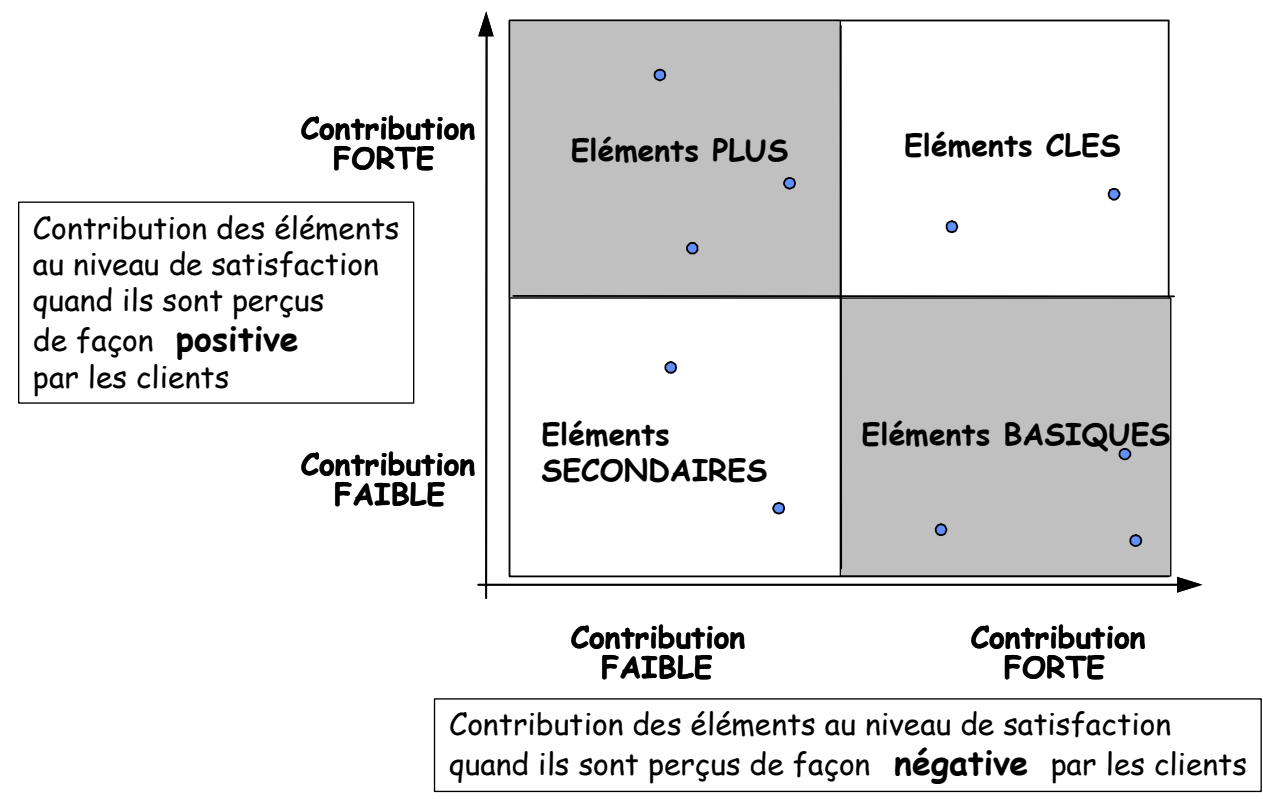

Ce modèle comporte deux axes, l'abscisse représente la contribution des éléments au niveau de satisfaction lorsqu'ils sont perçus de façon négative par les clients, l'ordonnée leurs contributions lorsqu'ils sont perçus de façon positive.

Dans le premier mode de contribution, le poids de certains éléments dans la satisfaction globale du client est stable, quelle que soit l'évaluation qui en est faite. Cette contribution stable peut être toujours forte, ce sont les éléments «Clés » ou toujours faible, les éléments « Secondaires ». 
Dans le second mode de contribution, les éléments contribuent à la satisfaction du client de façon fluctuante. Le poids des éléments dépend de leur niveau de performance perçu par le client. On distingue alors les éléments «Basiques» et les éléments «Plus »: lorsqu'un élément «Basique » est évalué négativement par le client, il influence fortement son niveau de satisfaction globale; une évaluation favorable, par contre, a peu d'impact sur sa satisfaction, c'est la moindre des choses. Les éléments «Plus» ont une logique de contribution inverse, s'ils sont bien perçus ils augmentent le niveau de satisfaction globale, sinon ils ont peu d'influence.

Ces modes de contribution semblent pouvoir s'appliquer à l'analyse de la satisfaction consécutive à la gestion d'une réclamation, et ce au regard des dimensions de la théorie de la justice.

\section{Methodologie}

Le terrain de l'étude a fait l'objet d'un contrat de recherche entre la CNAMTS (Caisse Nationale d'Assurance Maladie) et l'IAE de Lyon. L'étude a été réalisée auprès de professionnels de quatre Caisses Primaires d'Assurance Maladie (CPAM).

Cinq mille questionnaires ont été adressés à la cible des professionnels de santé. Sept-centvingt-cinq questionnaires ont été retournés dans les délais ${ }^{3}$. Les relations entre les CPAM et les assurés sociaux sont à distinguer de celles qu'elles entretiennent avec les professionnels de santé. En effet, ces derniers font l'objet d'une attention toute particulière, en bénéficiant, par exemple, de lignes téléphoniques dédiées et de personnels spécialement formés à leurs problématiques. Le système de management de la relation est construit sur le principe du portefeuille client afin d'assurer un suivi personnalisé.

Si ce terrain présente de nombreuses contraintes (secteur non concurrentiel des services publics, impossibilité de rupture, etc.), il présente néanmoins des avantages. La variable expliquée retenue dans cette étude est la satisfaction vis-à-vis de la gestion de la réclamation et non pas l'intention d'achat. De ce fait, l'impossibilité de rompre la relation ne pose pas de réel problème. De plus, la présente étude ne repose pas sur des scénarios ou des intentions de comportement mais sur des situations réelles de réclamations. En effet, le questionnaire débute par une question ouverte demandant si les professionnels de santé ont récemment eu

\footnotetext{
${ }^{3}$ Ce faible taux de retour s'explique en partie par trois faits : délai de réponse imparti très court, erreur de routage sur l'une des caisses et faiblesse de la qualification de l'échantillon qui a été constitué aléatoirement.
} 
un problème avec leur CPAM et si tel est le cas de décrire, de façon très détaillée, l'objet et la nature du problème les ayant conduits à faire une réclamation auprès de leur Caisse. La définition de ce que représente une réclamation est précisée dans la question (« demande d'explication, de rectification, de compensation et/ou d'amélioration de la qualité »). Un double codage ${ }^{4}$ des réponses permet d'extraire 342 réclamations dont 188 pour des problèmes de création/gestion des dossiers, 280 pour des problèmes de règlements financiers, 221 pour des problèmes qualité de service (rapidité, écoute, etc.). Le nombre de motifs est supérieur au nombre de réclamations car une réclamation pouvait faire l'objet de plusieurs motifs.

Les items du questionnaire relatifs à la gestion de la réclamation traitent les trois dimensions de la justice. Ces items sont issus de la synthèse réalisée par Tax et al. (1998). Ils ont été associés à des échelles de mesure en six points. Un item de contrôle a été utilisé pour s'assurer que la réclamation relatée correspondait bien à une source d'insatisfaction («quel était le niveau de votre insatisfaction concernant le problème rencontré $»)$. Les individus ne se déclarant pas insatisfaits sur cet item ont été écartés de l'échantillon. La satisfaction vis-à-vis de la gestion de la réclamation a été mesurée par un item unique : «Globalement, êtes-vous satisfait(e) du traitement de votre réclamation ».

Les mesures utilisées dans cette recherche sont toutes mono-item. Ce choix est dicté par deux types de considérations, de nature académique et managériale. La réflexion menée par Rossiter (2002) permet de penser que dans le cas de la mesure d'attributs simples, un item unique peut suffire, voire améliorer la qualité des résultats. En effet, l'auteur considère que, dans ces cas, la multiplication du nombre d'items implique la mesure d'attributs proches mais

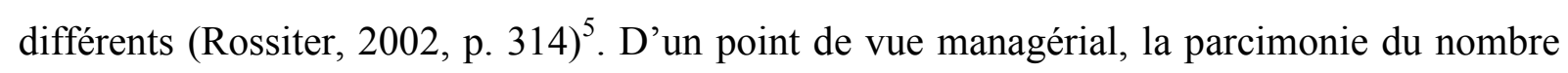
d'items s'explique également par les contraintes liées au partenariat avec la Caisse Nationale d'Assurance Maladie. En contrepartie, ce dernier donne accès à un «véritable » terrain d'étude et permet l'analyse de cas réels.

\footnotetext{
${ }^{4}$ Les divergences ont fait l'objet d'une recherche systématique de compromis.

${ }^{5}$ Les concepts («attributs ») utilisés dans cette recherche, comme par exemple, l'origine de la responsabilité perçue (locus) correspondent à ce cas de figure. Les éléments de justice relèvent d'une situation plus complexe. Certains d'entre eux sont des attributs simples, d'autres, comme par exemple la confiance, sont des attributs formatifs. Dans ce cas, Rossiter (2002) suggère qu'il peut être approprié de ne mesurer que les attributs les plus pertinents, tels que reconnus par des experts du domaine. Dans le cas présent, les mesures sont issues de la synthèse proposée par Tax et al. (1998).
} 


\section{La méthodologie du modèle Tétraclasse}

Le modèle «Tetraclasse » respecte les spécificités et la nature du construit de satisfaction, car les clients sont interrogés sur leur perception de différents éléments d'une expérience de service qu'ils viennent réellement de vivre. De plus les contributions sont déduites de l'analyse statistique et non déclarée par le client. La contribution des éléments à la satisfaction est calculée grâce à une analyse factorielle des correspondances d'un tableau de contingence comprenant :

- en colonne, les modalités d'un indice global de satisfaction réduit à deux classes (satisfaction positive / satisfaction négative) par une analyse de la médiane ;

- en ligne, les éléments susceptibles de jouer sur la satisfaction. Deux lignes ont été consacrées à chaque élément de l'expérience de service : l'une concerne son évaluation positive, l'autre son évaluation négative.

Le raisonnement suivant a été adopté : un seul axe factoriel suffit à expliquer $100 \%$ de la variance de l'ensemble étudié et peut être interprété comme un axe de satisfaction. Sur cet axe de satisfaction figurent deux modalités de satisfaction ainsi que deux modalités de chaque élément (évaluation positive et négative). Plus les éléments de service sont positionnés aux extrémités de cet axe, plus ils contribuent positivement ou négativement à la satisfaction du client. Un pliage de l'axe permet la visualisation de chaque élément étudié, par un point sur une carte: l'abscisse d'un point correspond à la contribution de l'élément au niveau de satisfaction lorsqu'il est évalué de façon négative. Son ordonnée est sa contribution au niveau de satisfaction lorsqu'il est évalué de façon positive. Les coordonnées de l'indice global de satisfaction permettent d'établir les frontières et de distinguer les quatre catégories d'éléments.

\section{Presentation des ReSUltats ET DiSCUSSION}

L'analyse des contributions des éléments de justice à la satisfaction vis-à-vis de la gestion des réclamations montre qu'il existe différentes logiques de contribution (cf. figure 2 et tableau 1 , ci-aprèł. 
Figure 2-Contribution des éléments de justice à la satisfaction vis-à-vis de la gestion de la réclamation

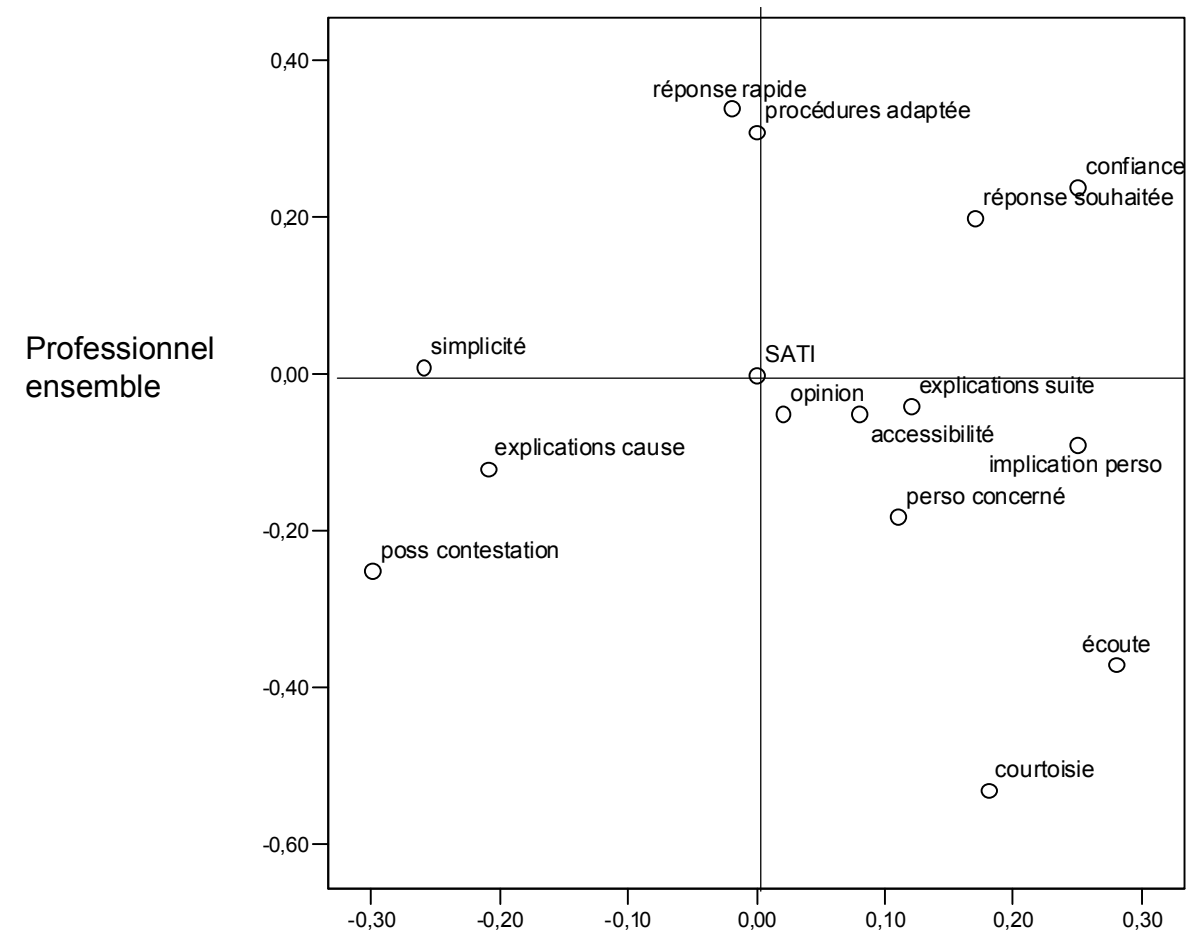

Tableau 1 - Récapitulatif des contributions des éléments de justice à la satisfaction vis-àvis de la gestion de la réclamation

\begin{tabular}{|c|c|c|c|c|c|}
\hline Justice distributive & Cat. & Justice procédurale & Cat. & Justice interactionnelle & Cat. \\
\hline $\begin{array}{l}\text { Confiance : La réponse faite } \\
\text { par le personnel de la CPAM } \\
\text { m'a inspiré confiance }\end{array}$ & C & $\begin{array}{l}\text { Rapidité : } \mathrm{La} \text { CPAM a } \\
\text { répondu rapidement à ma } \\
\text { réclamation }\end{array}$ & $\mathbf{P}$ & $\begin{array}{l}\text { Empathie : Le personnel } \\
\text { semblait vraiment concerné } \\
\text { par mon problème }\end{array}$ & B \\
\hline \multirow[t]{5}{*}{$\begin{array}{l}\text { Souhait : J'ai eu la réponse } \\
\text { que je souhaitais }\end{array}$} & $\mathrm{C}$ & $\begin{array}{l}\text { Flexibilité : } \mathrm{La} \text { CPAM a } \\
\text { adapté ses procédures pour } \\
\text { pouvoir régler mon problème }\end{array}$ & $\mathbf{P}$ & $\begin{array}{l}\text { Ecoute : Le personnel a } \\
\text { pris le temps d'écouter ma } \\
\text { réclamation }\end{array}$ & B \\
\hline & & $\begin{array}{l}\text { Contestation : } \mathrm{La} \text { CPAM } \\
\text { m'a donné la possibilité de } \\
\text { contester la réponse }\end{array}$ & $\mathbf{S}$ & $\begin{array}{llr}\text { Explications } & \text { (1) : } & \text { Le } \\
\text { personnel } & \text { m'a } & \text { bien } \\
\text { expliqué les } & \text { causes } & \text { du } \\
\text { problème } & & \end{array}$ & $\mathbf{S}$ \\
\hline & & $\begin{array}{l}\text { Accessibilité : Il a été facile } \\
\text { d'accéder à un moyen de } \\
\text { réclamation de la CPAM }\end{array}$ & B & $\begin{array}{lr}\text { Explications } & (2): \text { Le } \\
\text { personnel m'a } & \text { expliqué } \\
\text { clairement } & \text { comment } \\
\text { résoudre le problème }\end{array}$ & B \\
\hline & & $\begin{array}{l}\text { Simplicité : Les procédures } \\
\text { de réclamation sont simples }\end{array}$ & $\mathbf{P}$ & $\begin{array}{l}\text { Politesse : Le personnel a } \\
\text { répondu de manière } \\
\text { courtoise à ma demande }\end{array}$ & B \\
\hline & & $\begin{array}{l}\text { Participation : La CPAM a } \\
\text { pris en compte mon opinion } \\
\text { pour régler mon problème }\end{array}$ & B & $\begin{array}{l}\text { Implication : Le personnel } \\
\text { a réellement essayé de } \\
\text { régler mon problème }\end{array}$ & B \\
\hline
\end{tabular}

C pour Clé, B pour Basique, S pour Secondaire, P pour Plus. 
Les professionnels de santé sont particulièrement exigeants quant à la qualité de la gestion de leur réclamation. En effet, de nombreux éléments font partie de la catégorie basique soulignant qu'un bon niveau de performance doit être fourni.

Les éléments de justice distributive sont classés dans la catégorie «Clés ». La réponse apportée par l'organisation influence la satisfaction des professionnels quelle que soit la performance associée à cette réponse. Ces résultats confirment le caractère central de la réponse obtenue à l'issue du traitement de l'incident (Smith et al., 1999; Maxham et al., 2002b). L'entreprise doit donc porter une attention toute particulière au type de réponse à donner (compensation et/ou réparation par exemple). La cohérence entre la nature du problème rencontré par le professionnel de santé et la réponse apportée par l'organisation contribue toujours fortement au niveau de satisfaction.

Les modes de contributions des éléments de justice procédurale sont relativement hétérogènes. L'accessibilité du processus de réclamation et la prise en compte de l'opinion des professionnels de santé influencent le niveau de satisfaction uniquement lorsqu'ils sont évalués négativement (éléments «basiques»). Les efforts des CPAM pour faciliter la réclamation et l'écoute du point de vue des réclamants sont des éléments fondamentaux pour lesquels l'organisation doit fournir un niveau de performance en adéquation avec les attentes des professionnels. Au contraire, la rapidité, la capacité d'adaptation et la simplicité du processus contribuent fortement à la satisfaction lorsqu'ils sont évalués positivement (éléments «plus »). Les CPAM ont tout intérêt à fournir des niveaux de qualité élevés sur ces points lorsque l'investissement nécessaire ne paraît pas exagéré. Enfin, la possibilité laissée au professionnel de contester la réponse de l'organisation contribue plus faiblement à la satisfaction (élément secondaire). En d'autres termes, les professionnels de santé attendent une démarche pro-active. Ils ne considèrent pas la possibilité d'avoir « une seconde chance » comme un élément déterminants du traitement de la réclamation. Leur temps étant compté, il est important de répondre correctement à leurs attentes en première instance.

Cinq éléments de justice interactionnelle sur six appartiennent notamment à la catégorie éléments «Basiques ». Ce résultat complète les travaux de Tax et al. (1998) qui soulignent l'importance de l'interaction entre le personnel et les clients. Les professionnels ont un certain niveau d'attentes concernant l'écoute, la courtoisie et l'implication du personnel en contact. Ces éléments sont considérés comme faisant partie de la norme. Il s'agit donc de pré-requis dont le prestataire ne peut se dispenser. Il est donc important de respecter constamment ces 
standards relationnels. Les explications des causes du problème contribuent quant à elle relativement plus faiblement à la satisfaction des professionnels.

\section{CONCLUSION}

Rust et al. (1996) soulignent la nécessité et l'intérêt d'encourager les consommateurs insatisfaits à formuler une réclamation. Ils suggèrent également de récompenser les clients insatisfaits qui réclament et proposent une méthode pour évaluer la valeur des informations liées à cette réclamation. Ainsi l'entreprise a un double intérêt : conserver les gains futurs associés à la rétention des clients et améliorer la qualité de ses prestations. Néanmoins, ce raisonnement suppose que l'organisation propose un processus de gestion des réclamations efficace. En effet, si le client est prêt à laisser une seconde chance à l'organisation, la répétition des dysfonctionnements finit par être nuisible à l'organisation (Maxham et Netemeyer, 2002a). De nombreux travaux ont démontré l'intérêt des théories de la justice pour rééquilibrer l'échange entre un client déçu et une entreprise mise en défaut. Les résultats de ces études soulignent notamment l'importance des éléments distributifs (Smith et al., 1999 ; Maxham et al., 2002) et interactionnels (Tax et al., 1998). Mais ils ne permettent pas d'évaluer la contribution des éléments de gestion des réclamations selon la performance de l'entreprise.

Cet article apporte un éclairage sur les différentes logiques de contribution des éléments de justice à la satisfaction des clients suite à un incident de service. Le modèle tétraclasse de Llosa (1997) permet de considérer la variabilité des poids des éléments de justice. Les résultats de cette étude dans le cadre des relations entre les professionnels de santé et les Caisses Primaires d'Assurances Maladies confirment l'existence de différentes logiques de contribution. Certains éléments influencent le niveau de satisfaction seulement lorsqu'ils sont perçus de façon négative. Il s'agit notamment des éléments interactionnels. A l'inverse, les éléments procéduraux tels que l'adaptation, la rapidité et la simplicité des procédures contribuent à expliquer la satisfaction lorsqu'ils sont évalués de façon positive. D'autres éléments jouent sur la satisfaction quel que soit le niveau de performance, en particulier les éléments de justice distributive. Les résultats soulignent également que les démarches visant à expliquer les causes du problème ou à laisser la possibilité au client de contester à nouveau ne sont pas une priorité. 
Cette recherche reste cependant confrontée à un certain nombre de limites. Tout d'abord, le choix du terrain d'étude, les relations entre les professionnels de santé et leurs Caisses Primaires d'Assurance Maladie, peut être sujet à critique. Il s'agit là d'une organisation publique à but non commercial avec laquelle les professionnels de santé sont obligés de travailler. Cependant, les démarches d'amélioration de la qualité de service sont de plus en plus présentes dans ces organisations où l'usager devient progressivement client. De plus, le choix de ce terrain répond à un nécessaire arbitrage entre la liberté totale d'action du chercheur dans le cadre d'études reposant sur des scénarios auprès de populations choisies (dont Chébat et Slusarczyk -2005- soulèvent les limites) et les limites imposées par un partenariat donnant accès à des données relatives à des situations « réelles ».

D'autres limites, d'ordre méthodologiques, peuvent être soulevées. Par exemple, le classement de certains éléments à la frontière entre deux catégories de contribution est difficile. Il faut alors dépasser l'analyse quantitative et la stricte classification statistique et s'ouvrir à un regard plus qualitatif en intégrant notamment l'expérience des hommes de terrain.

Ces limites représentent autant de voies de recherche auxquelles d'autres pistes peuvent être ajoutées. D'une part, il est souhaitable de répliquer cette étude notamment dans un contexte marchand et librement concurrentiel. D'autre part, il serait intéressant d'étudier l'influence de variables individuelles - l'agressivité (Richins, 1983) ou la propension à réclamer (Seeking Redress Propensity) (Chébat et al., 2005),- ou situationnelles - par exemple, le type et l'ampleur du problème Levesque et McDougall, 2000) - sur la contribution des éléments de justice. Une autre voie de recherche consisterait à étudier le rôle de la responsabilité perçue de l'organisation sur la contribution des éléments de gestion de la réclamation à la satisfaction du client (Smith et al., 1999). En effet, c'est l'attribution de tout ou partie de la faute au prestataire qui conduit le client à formuler une réclamation (Day, 1984 ; Chébat et al., 2005). La question de la responsabilité paraît importante car elle peut influencer la perception de l'ampleur et de la nature des gains et des pertes pour chacune des parties. Enfin, seule la satisfaction vis-à-vis de la gestion de la réclamation a été étudiée dans cette recherche. Il serait pertinent d'étendre l'analyse à la satisfaction globale vis-à-vis de la relation ou encore la fidélité du client. L'étude du rôle de la responsabilité perçue de l'organisation sur l'ensemble de ces liens offre également de réelles perspectives de recherche. 


\section{BIBLIOGRAPHIE}

Adams J.S. (1965), Inequity in social exchange, in L. Berkowitz (Ed.), Advances in experimental social psychology, 2, 267-299.

Anderson R.E. (1973), Consumer Dissatisfaction : The Effect of Disconfirmed Expectancy on Perceived Product Performance, Journal of Marketing Research, 10, February, 38-44.

Audrain A.F (2004), Comment optimiser la satisfaction des consommateurs ? Une réponse par l'étude des relations attributs-satisfaction, Revue Française du Marketing, 197-2/5, mai 2004, 24-39.

Bartikowski B. (1999), La satisfaction des clients dans les services : Une vue situationnelle du poids fluctuant des éléments, W.P. n'542 IAE d'Aix-en-Provence, Février.

Bartikowski B. et Llosa S. (2001), De la théorie du poids fluctuant des éléments dans la satisfaction à la mesure. Comparaison empirique de quatre méthodes, Actes du 17ème congrès de l'Association Française du Marketing, éd. J. Brée, Deauville, CD-ROM.

Bartikowski B., Llosa S. (2004) Customer Satisfaction Measurement: Comparing Four Methods of Attribute Categorizations Service Industries Journal, 24, 4, 67-82.

Blodgett J. G., Hill D. J., Tax S. S. (1997), The Effects of Distributive, Procedural and Interactional Justice on Postcomplaint Behavior, Journal of Retailing, 73, 2, 185-210.

Boshoff C. and Leong J. (1998), Empowerment, attribution and apologising as dimensions of service recovery, An Experimental study, International Journal of Service Industry Management, 9, 1, 24-47.

Bowen D.E., Gilliland S.W. et Folger R. (1999), HRM and Service Fairness: How Being Fair with Employees Spills over to Customers, Organizational Dynamics, 27, 3, 7-23.

Brandt R.D. (1988), How service marketers can identify value-enhancing service elements, The Journal of Services Marketing, 2, 3, 35-41.

Brandt R.D. (1998), Attribute life cycle analysis - Alternatives to the Kano method, Actes de la conférence de l'ESOMAR, 171-183.

Cadotte E; Woodruff R; Jenkins R. (1987), Expectations and Norms in Models of Consumer Satisfaction, Journal of Marketing Research,_August,. 305-14.

Carman J. (1990), Consumer perceptions of service quality : an assessment of the SERVQUAL dimensions, Journal of Retailing, Spring, 33-55.

Chébat J.-C. et Slusarczyk W.(2005), How Emotions Mediate the Effects of Perceived Justice on Loyalty in Servce Recovery Situations : an Empirical Study; Journal of Business Research, 58, pp. 664-673.

Chébat J.-C., Davidow M. et Codjovi I. (2005), Silent Voices, Why some Dissatisfied Consumers Fail to Complain, Journal of Service Re search, 7, 4, 1-15. 
Clerfeuille F. et Poubanne Y. (2002), Contribution des éléments de satisfaction à la satisfaction, l'engagement et aux parts d'achats du consommateur : une étude exploratoire à partir du modèle tétraclasse. Actes du $18^{\text {ème }}$ Congrès de l'Association Française de Marketing, Lille.

Crié D. (2001), Un cadre conceptuel d'analyse du comportement de réclamation, Recherche et Applications en Marketing, 16, 1, 45-54.

Day R. (1984), Modeling Choices among Alternative Responses to Dissatisfaction with Durable Products, Advances in Consumer Research, 11, 496-99.

Dubois P.L. (1980), Le concept de Relief des attributs, Revue Francaise du Marketing, février,.19-30.

Folger R. et Konovsky M.A. (1989), Effects of procedural and distributive Justice on Reactions to pay Raise Decisions, Academy of Management Journal, 32, 1, pp. 115-130.

Folger R. et Cropanzano R. (1998), Organizational justice and human resource management, Sage Publications.

Grönroos C. (1993), Toward a Third Phase in Service Quality Research : Challenges and Future Directions, Advances in Services Marketing and Management, 2, 49-64.

Hart C.W.L., Heskett J.L. et Sasser W.E., (1990), The Profitable Art of Service Recovery, Harvard Business Review, 68, July-August, 148-156.

Herzberg F., Mausner B., Snyderman B. (1959) The motivation to work New York : John Wiley and sons, Inc.

Hocutt M. A., Chakraborty G., Mowen J. C. (1997), The Impact of Perceived Justice on Customer Satisfaction and Intention to Complain in a Service Recovery, Advances in Consumer Research, 24, 457-463.

Howard J. et Sheth J. (1969), The theory of buyer behaviour, New York John Wiley and sons.

Iacobucci D., Grayson K., Ostrom A. (1994), The Calculus of Service Quality and Customer Satisfaction : Theoretical and Empirical Differentiation and Integration, Advances in Services Marketing and Management : Research and Practice, 3, 1-67.

Kahneman D., Miller D. (1986), Norm Theory : Comparing Reality to its Alternatives, Psychological Review. 93, 2, 136-153.

Kano (1984), Myrioku-teki Hinshitu to Atari-mae Hinshitu, (Attractive quality and Must-be Quality). Hinshitu (Quality, the Journal of the Japonese Society for Quality Control ), 14, 239-48.

Léo P.Y., Philippe J. (2003) Positionnement concurrentiel des zones commerciales et satisfaction du consommateur,. Recherche et applications en Marketing, 18, 3, 45-63. 
Levesque T. J. et McDougall G. H. (2000), Service Problems and Recovery Strategies : An Experiment, Canadian Journal of Administrative Sciences, 17, 1, 20-37.

Lichtlé M.C., Llosa S., Plichon V. (2002), La contribution des éléments d'une grande surface alimentaire à la satisfaction du client, Recherche et Applications en Marketing, 17, 4, 23 34.

Lind E. A. et Tyler T. (1988), The social psychology of procedural justice, New York: Plenum.

Llosa S. (1997) L'analyse de la contribution des éléments du service à la satisfaction : Un modèle « tétraclasse » Décisions Marketing, 10, janv-avril, 81-88.

Maddox R.N. (1981), Two factors theory and consumer satisfaction : replication and extension, Journal of Consumer Research, 8, 1, 97-102.

Maxham J.G. III et Netemeyer R.G. (2002a), A Longitudinal study of Complaining Customers' Evaluations of Multiple Service Failures and Recovery Efforts, Journal of Marketing, 86, October, 57-71.

Maxham J.G. III et Netemeyer R.G. (2002b), Modeling customer perceptions of complaint handling over time : the effects of perceived justice on satisfaction and intent, Journal of Retailing, 78, 239-252.

Maxham J.G. III et Netemeyer R.G. (2003), Firms Reap What They Sow : The Effects of Shared Values and Perceived Organizational Justice on Customer Evaluations of Complaint Handling, Journal of Marketing, Janvier, 67, 1, 46-62.

Merdinger-Rumpler C. (2004), Contribution des elements de service à la satisfaction du patient hospitalise: le cas d'un service de chirurgie de court séjour, Actes $d u X X^{\text {ème }}$ congrès de l'Association Française de Marketing, 6 et 7 mai Saint Malo.

Myers J. et Alpert M. (1968), Determinant Buying Attitudes : Meaning and Measurement, Journal of Marketing, 32, October, 13-20.

Nicosia F. (1971), Processus de décision du consommateur, Paris, Dunod, Englewood-Cliffs, Prentice Hall.

Oliver R.L. (1980), A cognitive model of the antecedents and consequences of satisfaction decisions, Journal of Marketing Research, 17, 4, 460-469.

Poubanne Y., Clerfeuille F. et Chandon J-C. (2003), Variabilité catégorielle des éléments de service participant à la satisfaction des clients : une approche segmentaire Actes $d u 19^{\text {ème }}$ Congrès de l'Association Française de Marketing, 9 et 10 mai, Tunis.

Ray D. et Gotteland D. (2004) Mesurer d'asymétrie des impacts des facteurs de satisfaction : Compraison de la validité prédictive de cinq méthodes, Actes du XX'me congrès de l'Association Française de Marketing, 6 et 7 mai Saint Malo. 
Richins M. (1983), An analysis of consumer interaction styles in the marketplace, Journal of Consumer Research, 10, 73-82.

Rossiter J.R. (2002), The C-OAR-SE Procedure for Scale Development in Marketing, International Journal of Research in Marketing, 19, 305-335.

Rust R.T., Zahorik A.J. et Kenningham T.L. (1996), Service marketing, New York : Harper Collins.

Sabadie W. (2000), Les apports des théories de la justice au marketing des services, Congrès International de l'Association Française du Marketing, Montréal, 133-147.

Schneider B. et Bowen D. (1995), Winning the service game, Harvard Business Edition .

Seiders K. et Berry L. L. (1998), Service fairness : what it is ans why it matters, Academy of Management Executive, 12, 8-20.

Silvestro R.et Johnston R. (1992), The determinants of service quality: hygiene and enhancing factors, Quality in Services Conferences, The Business Research Institute at John's University, New York.

Smith A. K., Bolton R.N. et Wagner J. (1999), A Model of Customer Satisfaction with Service Encounters Involving Failure and Recovery, Journal of Marketing Research, vol. 36, August, 356-372

Smith W., Weatherly K. et Tansik D. (1992), A customer based service quality study : use of the sorting/cluster analysis methodology, $2^{\text {ème }}$ Séminaire International de Recherche en Management des Activités de Service, La Londe les Maures.

Swan J.E. et Combs L.J. (1976), Product performance and consumer satisfaction : a new concept, Journal of Marketing, 40, 2, 25-33.

Tax S.T., Brown S.W. et Chandrashekaran M. (1998), Customer evaluations of service complaint experiences implications for relationship marketing, Journal of Marketing, 62, 60-67.

Tissot M. \& Chandon J.-L. (2004), Modeling the Service Recovery Impact on Dissatisfied Consumers: The Moderating Impact of «Estimated Knowledge », Proceedings of the International Research Seminar in Service Management, La Londe les Maures, Université d'Aix en Provence, June, 765-787.

Vernette E. (1986), Comparaison des méthodes d'identification des critères de choix d'un produit, Thèse pour le Doctorat d'Etat es Sciences de gestion, Université de Paris X Nanterre

Westbrook R.A. (1987), Product/consumption-based affective responses and post purchase processes, Journal of Marketing Research, 24, 3, 258-270.

Woodruff R.B., Cadotte. B.E. et Jenkins R.L. (1983), Modeling consumer satisfaction processes using experience-based norms, Journal of Marketing Research, 20, 3,296-304. 